\title{
Nursing infectious disease: a history with three lessons*
}

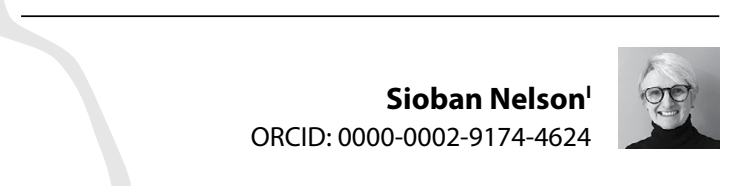

'University of Toronto, Faculty of Nursing. Toronto, Canada.

How to cite this article:

Nelson S. Nursing infectious disease: a history with three lessons. Rev Bras Enferm. 2021;74(3):e740301. https://doi.org/10.1590/0034-7167.2021740301
* This paper won first prize in the COVID-19 history lessons for managers essay competition submission 21 April, 2020. It was first published as "Nursing infectious disease: a history with three lessons". ZUG Zeitschrift für Unternehmensgeschichte, 2020, 65(2): 305-309. September 2020, De Gruyter. DOI: 10.1515/ zug-2020-0005.
There is something uncanny about the fact that the bicentenary of the birth of Florence Nightingale and the WHO Year of the Nurse and Midwife was launched with a pandemic and the back-to-the-future message of "wash your hands"(1). In Notes on Nursing, her enormously popular household primer on care of the sick, Nightingale was unequivocal:"Every nurse ought to be careful to wash her hands very frequently during the day. If her face too, so much the better"(2). For trained nurses Nightingale went even further, boil "everything, even the surgeon" she joked ${ }^{(3)}$. Nightingale would approve, I suspect, of the public's rush on bleach and cleaning products, inasmuch as it indicates that the message of cleanliness appears to have hit home at long last. But there is no doubt she would be profoundly shocked by the infection rate of health workers, as well as the unknown numbers of hospital patients and residents of long-term care for whom the source of their COVID-19 infection was none other than the nurses, doctors and personal support workers who cared for them ${ }^{(4)}$. And while the lack of PPE is clearly a major cause of infection, PPE on its own is insufficient to ensure health care workers will neither contract nor spread the disease. In what follows, I put forward three areas where nursing history can offer, in the words of Nightingale, "suggestions for thought" to protect and support staff from nosocomial infection and to aid recovery post COVID-19(5).

First lesson: back to basics. By the end of the nineteenth century and well into the mid-twentieth century, the care of patients with infectious diseases was a major element of nursing work in general adult and paediatric hospitals, in special infectious disease hospitals, as public health nurses and as private duty nurses in the home. All nurses underwent rigorous training in order to be able to apply the principles of infection control in whatever context they found themselves. The instructions in the Manual for Fever Nursing published in New York in 1904 included a chapter on The Sick Room and its Furniture. Addressing the issue of protective clothing, what we know today as personal protective equipment or PPE, it declared that in the case of diphtheria: "The nurse and the physician should wear, while in the sick room, a gown which covers the clothing entirely. This should be kept outside the apartment and sterilized directly after use. If the patient, while the throat is being examined, should cough in the examiner's face, the latter should wash the face and hair in soap and water followed by 1:1,000 mercury bi-chloride solution. The hands must always be sterilized upon leaving the sick room"(6).

The rigid discipline associated with the military style charge nurses or "sisters" of the post WWII era faded with the rise of informality that came during the 70s and 80s. Beyond a change in manners and mores, the latter decades of the twentieth century witnessed a rise in patient complexity and the need for better educated nurses, increased multi-disciplinary care, and a precipitous decline in average length of stay - all of which heralded the end of the age of the matron and charge nurse. But there was a time when nurses would be hauled over the coals for wearing nail polish, or draping their stethoscope around their neck, or a thousand other infringements of the dress and conduct code. Underpinning this regimentation was an 
orientation, a way of thinking, that identified objects with the capacity to become fomites and carry infectious material around the hospital or to and from the home. Most health workers today have a relaxed approach to their hair, or their pens or pockets, something that would have been unacceptable in the past. It is paramount to their safety and to the safety of others that staff re-learn, or perhaps learn for the first time, the mindset of the pre-vaccination pre-antibiotic era, to think of every single item of clothing and object on their person, and every item in a patient's room or piece of hospital equipment as potential carriers of COVID-19. Such a shift in thinking will also necessitate a change in the way space is ordered in the hospital and the free movement of health personnel throughout the institution.

The second lesson: innovation in the face of a new disease. What new practices does the care of COVID-19 patients entail? Prior to the introduction of vaccines and antibiotics there was no cure for infectious illness and no preventive measures other than hygiene and strict isolation of contacts. Isla Steward, the indominable matron of St Bartholomew's Hospital London for decades was early in her career placed in charge of the London Thames River Smallpox river camp hospital. Over the course of the epidemic $1884-85$ she presided over a staff of 100 nurses who cared for 1,800 patients under canvas with a mortality rate of only 17 per cent as opposed to the expected 30-40 per cent ${ }^{(7)}$. After the epidemic Stewart explained that doctors believed small pox was a «nurses» disease because without a cure the care and comfort of the patient was predominantly "the nurse's prerogative" ${ }^{\prime(7)}$. The nursing care in infectious disease was supportive: the management of fever (a huge speciality area of practice) and symptom management such as throat lavage in diphtheria, skin care in small pox or measles, pain management in typhoid. The development of best practice in COVID-19 care, in the support and comfort of dying patients, and in care of those at home or in long-term care will require a multi-disciplinary effort and constitute a major advance in the compassionate and effective management of this disease.

When HIV/AIDS swept the world, generating stigma and fear, the entire health care industry shifted to a universal precautions or standard measures approach to handling bodily fluids in order to protect health workers ${ }^{(8)}$. New tools for blood collection and shifts in everyday practices that involved the handling of sharps followed. Gloves, masks, eye protective equipment and disposable gowns were produced in vast quantities and became part of routine care. As the evidence on COVID-19 grows it is becoming increasingly clear that asymptomatic and pre-symptomatic individuals are a major cause of infection. The adoption of universal precautions against droplet infection will entail a major shift in the organization of care and a total revision of standard practice ${ }^{(9)}$. Learning from the introduction of universal precautions for bloodborne products and bodily fluids in the 1980s and 90s, and the challenges with compliance to complete overhauls in procedures offers managers some guidance in the implementation of new protocols and new equipment that will surely follow COVID-19.

Third lesson: staffing in the new era of infectious disease. What will staffing hospitals, home care and long-term care look like in the years ahead? The recent report on nursing from the WHO stressed the inequitable distribution of nurses globally calling for the creation of six million new nursing jobs by 2030 . And this is before the arrival of COVID-19(10). Nursing recruitment has long experienced boom bust cycles but the impact of infectious disease can be found in the case of the 1950s recruitment to the NHS. Longstanding shortages of nursing staff along with the creation of the NHS combined to require aggressive recruitment of nurses from within the UK and Ireland and the Caribbean. Staff coming to urban areas from rural areas were found to be particularly vulnerable to infectious illness. Immigrant nurses, such as the first wave Caribbean nurses too had heightened vulnerability ${ }^{(7)}$. The management of staff with varying levels of vulnerability to COVID-19 will require careful oversight for years to come.

Finally, under the headline of "Good news for tired-out members", the British College of Nursing Bulletin of October 1920 announced the opening of a seaside cottage on the Isle of Wight for the convalescence of sick nurses ${ }^{(11)}$. Modern medicine is largely premised on aggressive management and the assumption of rapid recovery. For those who survive a serious infection of COVID-19, a disease where relapse or reinfection could be possible, it may be necessary to consider how rehabilitation and recuperation can be supported. Following each world war, the overwhelming number of disabled and incapacitated veterans led to the creation of the rehabilitation movement, new fields of medicine and state involvement in long-term support of veterans. As Julie Anderson points out in her work on war and disability in Britain, this movement was characterized by significant gender inequities ${ }^{(12)}$. Given what we are currently experiencing with the disproportionate burden of COVID-19 on racialized and impoverished communities, we will need to ensure any ongoing support of COVID-19 survivors, be they patients, health workers or both, does not heighten these disparities. We may even wish to consider a return to the now archaic notion of convalescent homes. Is it too much to hope that the current public fervor in support of front-line health workers, especially nurses, might even translate into donations for seaside cottages at nurses' disposal to ensure full return of a healthy workforce in the post pandemic world? One can live in hope!

\section{REFERENCES}

1. World Health Organization (WHO). Year of the Nurse and Midwife Campaign [Internet]. 2020[cited 2020 Jun 04]. Available from: https:// www.who.int/news-room/campaigns/year-of-the-nurse-and-the-midwife-2020

2. Nightingale F. Notes on nursing. what it is and what it is not [Internet]. New York 1989[cited 2020 Jun 04]. 80p. Available from: http:// sciencegraph.org/documents/Notes-on-nursing.pdf

3. Nelson S, Rafferty AM. Notes on Nightingale: the influence and legacy of a nursing icon. New York; Cornell University Press: Ithica. 2010. 
4. International Council of Nursing. See the ICN statement on the issue [Internet]. 2020[cited 2020 Jun 04]. Available from: https://www.icn.ch/ news/icn-says-worldwide-death-toll-covid-19-among-nurses-estimated-100-may-be-far-higher

5. Nightingale F. Florence Nightingale's Suggestions for Thought: collected works of Florence Nightingale. McDonald Lynn, 11:Waterloo; 2008. 184 p.

6. Wilcox RW. A Manual of Fever Nursing: lectures on fever nursing which were delivered in substance to the nurses of St Mark's hospital during the season of 1903-4 [Internet]. Philadelphia; 1904[cited 2020 Jun 04]. p157-160. Available from: https://archive.org/details/ manualoffevernu00wilc/page/228/mode/2up

7. Currie M. Fever Hospitals and Fever Nurses: a British Social History of Fever Nurses. London: Routledge; 2005,128.

8. SE Beekmann, Vlahov D, Koziol DE, McShalley ED, Schmitt JM, Henderson DK. Temporal association between implementation of universal precautions and a sustained, progressive decrease in percutaneous exposures to blood. Clin Infect Dis. 1994;4(4):562-9. https://doi. org/10.1093/clinids/18.4.562

9. Wu S, Li L, Wu Z, Cao H, Lin C, Yan Z, Jia M, Cui H. Universal precautions in the era of HIV/AIDS. AIDS Behav. 2008;12(5):806-14. https://doi. org/10.1007/s10461-007-9278-8

10. World Health Organization (WHO). State of the World's Nursing Report [Internet]. 2020[cited 2020 Jun 04]. Available from: https://www.who. int/publications-detail/nursing-report-2020

11. Royal College of Nursing. Florence Nightingale. Bull[Internet]. 1920[cited 2020 Jun 04];1(4). Available from: https://rcn.access.preservica. com/uncategorized/IO_445a0f62-1bea-47b7-a0a0-bf35a607c765/

12. Anderson J. War disability and rehabilitation in Britain: soul of the nation. Manchester: University Press; 2011. 